\section{Kidney \\ Blood Pressure Research}

\title{
Evaluation of Absolute Serum Creatinine Changes in Staging of Cirrhosis-Induced Acute Renal Injury and its Association with Long-term Outcomes
}

\author{
Fangfang Zhou ${ }^{\mathrm{a}}$ Qun Luo ${ }^{\mathrm{a}}$ Lina Han ${ }^{\mathrm{a}}$ Huadong Yan ${ }^{\mathrm{b}}$ Wenhong Zhou \\ Zemin Wang ${ }^{\mathrm{a}}$ Yumei $\mathrm{Li}^{\mathrm{a}}$
}

${ }^{a}$ Department of Nephrology; ${ }^{b}$ Department of Liver Diseases, Ningbo No. 2 Hospital, Ningbo University School of Medicine, Ningbo city, Zhejiang province, P.R. China

\section{Key Words}

Acute kidney injury $\bullet$ Cirrhosis $•$ Classification $\bullet$ Creatinine $\bullet$ Mortality

\begin{abstract}
Background/Aims: To assess the prognostic accuracy of absolute serum creatinine $(\mathrm{s} C \mathrm{r})$ changes ('Delta-sCr') on the long-term outcomes in cirrhotic patients, and evaluate the performance of the 'Delta-sCr' approach to stage acute kidney injury (AKI), compared with the Kidney Disease Improving Global Outcomes (KDIGO) criteria. Methods: We conducted a retrospective analysis of 333 hospitalized patients. We classified AKI stages using two methods: 1) KDIGO AKI criteria; 2) 'Delta-sCr' system, defined by the difference between the baseline and the peak $\mathrm{s} C r$ value during the hospitalization. The end point was the hazard of 1-year death. Results: The prevalence of AKI in cirrhotic patients was $18.01 \%$ by the KDIGO criteria, and $25.22 \%$ by the 'Delta-sCr' system. On multivariable Cox hazard analysis, both of the two methods were independent predictive factors of death ('Delta-sCr' system: $\mathrm{OR}=2.911$, $\mathrm{p}<0.001$ ), (KDIGO criteria: OR=2.065, $\mathrm{p}<0.001)$. However, the 'Delta-sCr' system provided a modest improvement in classification over the KDIGO criteria with a net reclassification improvement (NRI) of $28.7 \%(p<0.001)$ and integrated discrimination improvement (IDI) of $7.5 \%$ $(p=0.03)$. And the predictive value of the 'Delta-sCr' system could be significantly improved $(p=0.006)$, when combined with age and MELD score. Conclusion: The Delta-sCr is associated with the 1-year mortality. And the 'Delta-sCr' system may optimize the discrimination of risk prediction.
\end{abstract}




\section{Kidney Blood Pressure Research}

\section{Introduction}

Acute kidney injury (AKI) is a common complication in cirrhotic patients and is associated with a poor prognosis independently $[1,2]$. Approximately $20 \%$ of hospitalized patients with cirrhosis develop renal failure [3]. And a meta-analysis showed AKI predicting a 7-fold increased risk of death in cirrhotic patients [4]. With the improved understanding of renal failure in cirrhosis, the definition of renal failure has been challenged. Recently, a working group composed of members of the International Ascites Club (IAC) and the Acute Dialysis Quality Initiative (ADQI) reached the final proposal for the diagnosis of cirrhosisAKI, named IAC-AKI, which is defined that any patients with cirrhosis and an increase in serum creatinine ( $\mathrm{sCr}$ ) by either $26.5 \mu \mathrm{mol} / \mathrm{L}$ within $48 \mathrm{~h}$ or by $50 \%$ from baseline within 7 days, would be classified as having AKI, abandoning the threshold of $\mathrm{sCr} \geq 133 \mu \mathrm{mol} / \mathrm{L}$ in the traditional criteria [5].

However, as to the classification system specific to cirrhosis-AKI, there is still an area of great controversy so far [6]. Several prospective studies have demonstrated that the risk of cirrhotic, patient mortality was directly related to the patients severity of AKI [7-9]. Hence, an exact AKI staging system, rather than a simple binary descriptor, is more crucial to help the physicians to predict the clinical outcome and decide on the intensity of monitoring and consequently, the most appropriate treatments for patients with cirrhosis. Existing widely used AKI staging systems in nephrology include the RIFLE (Risk-Injury-Failure-Loss-End stage renal disease) criteria, the AKIN (Acute Kidney Injury Network) criteria, and now the KDIGO (Kidney Disease Improving Global Outcomes) criteria, which are all based on the relative changes in sCr. Recently, some studies proposed to stage AKI using absolute increases in $\mathrm{sCr}$, which indicated a promising alternative to the KDIGO criteria for characterizing the severity of AKI [10-12]. Those studies were mostly validated amongst general hospitalized patients, but its application specific to cirrhotic patients is less certain.

Our study was performed to access the prognostic accuracy of absolute sCr changes ("Delta-sCr") on the long-term outcomes(1-year mortality) in patients with cirrhosis, and evaluate the performance of the "Delta-sCr" approach to stage AKI, compared with the KDIGO criteria, using the relative increases in $\mathrm{sCr}$.

\section{Materials and Methods}

\section{Study Design and Patients}

The study was conducted retrospectively, including cirrhotic patients admitted to the Department of Liver Diseases of Ningbo N0.2 Hospital, a 2000-bed, tertiary care and university affiliated hospital, between January 2013 and December 2014. Inclusion criteria included (1) patients age $\geq 18$ years with diagnosis of liver cirrhosis of any etiology. And the diagnosis of cirrhosis was established by clinical, biochemical, ultrasonographic and endoscopic findings; (2) patients with at least one outpatient sCr measurement within 1 year before hospitalization, or $>1$ measurement during the hospitalization. The criteria for exclusion included (1) previous liver or kidney transplantation; (2) hepatocellular carcinoma outside Milan criteria or advanced non-hepatic neoplasia; (3) advanced chronic kidney disease (baseline sCr $>353.6 \mu \mathrm{mol} / \mathrm{L}$ ) acute or chronic renal replacement therapy before admission; (4) patients who had incomplete sCr data. Patients' electronic medical records were reviewed including basic data such as demographics, laboratory results, and comorbidities at the time of admission were collected. Only Data from the initial admission was analyzed exclusively for patients with multiple admissions. MELD and Child-Pugh score were also calculated from the time of admission.

Baseline sCr was defined as the most recent and lowest value during the 7 days to 1 year preceding hospitalization (in 232/333 patients, 69.67\%) or the sCr value at admission (in 101/333 patients, 30.33\%), if the former was not available. And the peak $\mathrm{sCr}$ was defined as the highest $\mathrm{sCr}$ value reached during hospitalization. 


\section{Kidney \\ Blood Pressure Research}

We classified AKI stages using two methods: 1) KDIGO AKI staging criteria (KDIGO criteria) [13], defined at the time of first fulfillment: Stage 1: increase in $\mathrm{sCr} \geq 26.5 \mu \mathrm{mol} / \mathrm{L}$ within 48 hours or sCr increase 21.5-1.9times baseline within 7days; Stage 2: sCr increase 2-2.9times baseline; Stage 3: sCr increase $\geq 3$ times baseline. In the criteria, the use of urine output as one of the criteria has been removed since it is inaccurate for diagnosis in patients with cirrhosis; 2) the 'Delta-sCr' system, which was defined by the difference between the baseline and the peak sCr value. Cut-offs were determined based upon the KaplanMeier survival analysis to optimally differentiate survival function for each stage [12]: Stage 1, Delta-sCr: 26.5-132.6 $\mu \mathrm{mol} / \mathrm{l}$ (0.3-1.5mg/dl), Stage 2, Delta-sCr: 132.7-221.0 $\mathrm{mol} / \mathrm{l}$ (1.5-2.5mg/dl) and Stage3, Delta$\mathrm{sCr}>221.0 \mu \mathrm{mol} / \mathrm{l}(2.5 \mathrm{mg} / \mathrm{dl})$.

Patients were followed up at an outpatient clinic or via telephone contact (records were collected in the electronic medical system). The end point (for 1 year) was the hazard of death. For those patients whose lengths of follow-up were more than 1 year, the survival time was censored at 365 days after admission.

The study protocol was approved by the Ethics Committee of Ningbo NO.2 Hospital according to Institutional guidelines. Given the retrospective nature of the study, we were granted a waiver of informed consent.

\section{Data Analysis}

Continuous variables were reported as mean and standard deviation or median, minimum and maximum. Categorical variables were reported as count and percentage in each category. Univariate logistic analyses were conducted to identify potential predictors of end point. Multivariate, logistic, and Cox regression models were performed by a backward elimination technique to explore the independent prognostic value of predictors. To prevent multicollinearity, we created other models for multivariate analysis, excluding those variables that are computed from other variables, such as MELD score or ChildPugh score. Since the factors peak sCr, and Delta sCr were highly correlated with AKI stages, we did not put them into the same model. Moreover, multivariate analysis was repeated with either of the AKI staging method to avoid multicollinearity.

1-year survival probability curves of different stages of AKI were calculated with the Kaplan-Meier method and compared with log-rank test. The prognostic values of the two methods were demonstrated by using the area under ROC curve (AUC) evaluation metric. DeLong's method was used for ROC curve comparison. The predictive values for 1-year mortality of "Delta-sCr" system was compared with KDIGO criteria using the net reclassification improvement (NRI) and integrated discrimination improvement (IDI). The confidence interval was accepted as $95 \%$ and p values equal or smaller than 0.05 were accepted as statistically significant. SPSS Version 22.0 (SPSS, Inc, an IBM Company, Chicago, Illinois, USA) and R i386 3.3.0 software (for the calculation of NRI and IDI) were used for statistical analysis.

\section{Results}

Demographic Characteristics and Clinical Features

A total of 333 cirrhotic patients were enrolled in the present study. The median age was $55.68 \pm 12.56$ years, and $63.06 \%(n=210)$ of the patients were male. The most common etiology of cirrhosis was HBV infection $(n=232,69.7 \%)$, followed by Miscellaneous $(n=40$, $12 \%)$, Alcohol $(n=30,9 \%)$, Cholestasis $(n=17,5.1 \%)$, and Autoimmune $(n=14,4.2 \%)$. Mean MELD and Child-Pugh scores on admission were 11.58 and 9, respectively. The prevalence of AKI was found to be $18.01 \%$, defining by KDIGO criteria, and $25.22 \%$ by the "Delta-sCr" system. 24 patients with mild renal injury were detected by the "Delta-sCr" system, whose $\mathrm{sCr}$ increase $\geq 26.5 \mathrm{umol} / \mathrm{l}$ beyond a $48 \mathrm{~h}$ time period, but $<1.5$ folds in 7 days, without reaching the KDIGO criteria. Among them, three patients (12.5\%) died in the follow-up.

After 1-year follow-up, 303 patients were alive and 31 dead. No patient was lost to follow-up. The cohort was divided into two groups based on the 1-year mortality rate of all the patients. The patients of the 'non-survivor group' were older, with higher severity of cirrhosis, higher rates of complications of infection and chronic kidney disease (CKD). AKI was developed much more in the 'non-survivor group'. (Table 1) 


\section{Kidney Blood Pressure Research}

Table 1.Baseline characteristics and Univariate analysis for factors associated with 1-year mortality

\begin{tabular}{|c|c|c|c|c|c|}
\hline & $\begin{array}{c}\text { Total } \\
(\mathrm{n}=333)\end{array}$ & $\begin{array}{l}\text { Survivor } \\
(\mathrm{n}=302)\end{array}$ & $\begin{array}{l}\text { Non-survivor } \\
\quad(\mathrm{n}=31)\end{array}$ & $\begin{array}{c}\text { Survivor } \\
\text { VS. } \\
\text { Non-survi } \\
\text { vor } p \\
\end{array}$ & $\begin{array}{c}1 \text {-year } \\
\text { mortality } \\
\text { Univariate } p\end{array}$ \\
\hline Age,yr & $55.68 \pm 12.56$ & $54.87 \pm 0.72$ & $63.80 \pm 1.75$ & $<0.001$ & $<0.001$ \\
\hline Sex,Male & $210(63.06 \%)$ & $188(62.05 \%)$ & $22(73.33 \%)$ & 0.325 & 0.411 \\
\hline Etiology & & & & 0.175 & 0.327 \\
\hline HBV & $232(69.7)$ & $210(69.3)$ & $20(66.7)$ & & \\
\hline Alcohol & $30(9.0)$ & $23(7.6)$ & $7(23.3)$ & & \\
\hline Autoimmune & $14(4.2)$ & $13(4.3)$ & $1(3.3)$ & & \\
\hline Cholestasis & $17(5.1)$ & $17(5.6)$ & $0(0)$ & & \\
\hline Miscellaneous & $40(12)$ & $38(12.5)$ & $2(6.7)$ & & \\
\hline Child-Pugh Score & $9[5,15]$ & $9[5,15]$ & $12[6,15]$ & $<0.001$ & $<0.001$ \\
\hline MELD Score & $11.58[1.75,35.02]$ & $10.67[1.75,28.26]$ & $16.82[7.71,35.02]$ & $<0.001$ & $<0.001$ \\
\hline MAP,mmHg) & $92.66[82,100]$ & $93.33[53.33,128.67]$ & $87.33[54,134.67]$ & 0.066 & 0.055 \\
\hline WBC count,$\times 10^{9} / \mathrm{L}$ & $4.10[0.90,27.90]$ & $4[0.90,27.60]$ & $5.90[1.50,24.50]$ & $<0.001$ & $<0.001$ \\
\hline Platelet count,$\times 10^{9} / \mathrm{L}$ & $74[3,358]$ & $74[16,358]$ & $75[3,168]$ & 0.673 & 0.690 \\
\hline INR & $1.39[0.80,4.53]$ & $1.37[0.80,2.90]$ & $1.50[1.18,4.53]$ & $<0.001$ & $<0.001$ \\
\hline Tbil, $\mu \mathrm{mol} / \mathrm{L}$ & $36.30[2.60,523.60]$ & $32.30[2.60,523.60]$ & $60.10[13.40,444]$ & 0.031 & 0.021 \\
\hline Alb,g/L & $29.10 \pm 6.10$ & $29.11 \pm 0.34$ & $27.86 \pm 1.34$ & 0.224 & 0.285 \\
\hline ALT,g/L & $34[3,758]$ & $35[3,758]$ & $31[3,446]$ & 0.075 & 0.089 \\
\hline $\mathrm{TBA}, \mu \mathrm{mol} / \mathrm{L}$ & $55.90[0.20,390.10]$ & $53[0.20,348.80]$ & $80[7.70,390.10]$ & & 0.155 \\
\hline Serum Sodium ,mmol/L & $138.43 \pm 5.75$ & $138.92 \pm 0.29$ & $133.51 \pm 1.59$ & $<0.001$ & $<0.001$ \\
\hline $\mathrm{CRP}, \mathrm{mg} / \mathrm{L}$ & $5.76[0.03,324.16]$ & $5.24[0.03,324.16]$ & $12.93[0.28,203.80]$ & 0.001 & $<0.001$ \\
\hline SBP & $53(15.91)$ & $45(14.85)$ & $8(26.67)$ & 0.195 & 0.092 \\
\hline Other Bacterial Infection & $75(22.52)$ & $60(19.80)$ & $15(50.00)$ & 0.001 & $<0.001$ \\
\hline DM & $60(18.02)$ & $52(17.16 \%)$ & $8(26.67)$ & 0.307 & 0.197 \\
\hline Hypertension & $47(14.11)$ & $40(13.20 \%)$ & $7(23.33)$ & 0.244 & 0.129 \\
\hline Baseline $\mathrm{sCr}, \mu \mathrm{mol} / \mathrm{L}$ & $70.9[20.30,240.10]$ & $68.10[20.30,240.10]$ & $88.74[64.50,152.10]$ & $<0.001$ & $<0.001$ \\
\hline Peak sCr, $\mu \mathrm{mol} / \mathrm{L}$ & $76.60[42.60,639.80]$ & $74.70[42.60,409.70]$ & $228.40[71.80,639.80]$ & $<0.001$ & $<0.001$ \\
\hline Delta-sCr, $\mu \mathrm{mol} / \mathrm{L}$ & $7.2[0.10,543]$ & $5.80[0.10,291.60]$ & $54.80[5.30,543]$ & $<0.001$ & $<0.001$ \\
\hline CKD & $24(7.20)$ & $21(6.93)$ & $3(10.00)$ & 0.041 & 0.049 \\
\hline KDIGO criteria & & & & $<0.001$ & $<0.001$ \\
\hline Stage 0 & 273(81.98) & $264(87.41)$ & $9(29.03)$ & & \\
\hline Stage 1 & $32(9.60)$ & $23(7.62)$ & $9(29.03)$ & & \\
\hline Stage 2 & $16(4.80)$ & $10(3.31)$ & $6(19.35)$ & & \\
\hline Stage 3 & $12(3.60)$ & $5(1.66)$ & $7(22.58)$ & & \\
\hline Delta-sCr system & & & & $<0.001$ & $<0.001$ \\
\hline Stage 0 & $249(74.77)$ & 243(80.46) & $6(19.35)$ & & \\
\hline Stage I & $60(18.02)$ & $50(16.56)$ & $10(32.26)$ & & \\
\hline Stage II & $14(4.20)$ & $8(2.65)$ & $6(19.35)$ & & \\
\hline Stage III & $10(3.00)$ & $1(0.33)$ & $9(29.03)$ & & \\
\hline \multicolumn{6}{|c|}{$\begin{array}{l}\text { Values are given as numbers(n), percentage(\%), means } \pm \text { SD. or median [min,max].HBV, Hepatitis B virus; MELD, Model for } \\
\text { End Stage Liver Disease; MAP, Mean arterial pressure; WBC, White blood cell; INR, International normalized ratio; Tbil, } \\
\text { Total bilirubin; Alb, Serum Albumin; ALT, Alanine aminotransferase; TBA, Total bile acid; CRP, C-reactive protein; SBP, } \\
\text { Spontaneous bacterial peritonitis; DM, Diabetes mellitus; sCr , serum creatinine; CKD, Chronic Kidney Disease; KDIGO, } \\
\text { Kidney Disease Improving Global Outcomes. }\end{array}$} \\
\hline
\end{tabular}

\section{The End Point}

At the end of the 1-year follow-up period, a total of 31 patients (9.31\%) had died. 8 patients died during hospitalization whereas 23 patients died after the discharge from the hospital. A univariate analysis of survival demonstrated that variables related with age $(p<0.001)$, Child-Pugh score $(p<0.001)$, MELD score $(p<0.001)$, WBC count $(p=<0.001)$, INR $(p<0.001)$, Tbil ( $p=0.021)$, Serum Sodium $(p<0.001)$, CRP $(p<0.001)$, all the renal parameters including sCr (Baseline $\mathrm{sCr}(p<0.001)$, Peak sCr $(p<0.001)$, and Delta $\mathrm{sCr}(p<0.001))$, AKI stages according to KDIGO criteria $(p<0.001)$ or 'Delta-sCr' system $(p<0.001)$ and CKD $(p=0.049)$, as well as Other Bacterial Infection $(p<0.001)$ were associated with mortality. 


\section{Kidney Blood Pressure Research}

Several multivariate Cox regression models were performed to exclude multicollinearity. In Model 1 including individual variables and excluding liver scores and AKI classification, the independent predictive factors of survival were: Age, WBC count, and INR. When liver scores were included in the analysis (Model 2), the independent predictive factors were: age and MELD score. And either of the two staging methods of AKI was independent predictive factor of survival (KDIGO:
Table 2. Independent predictors of 1-year mortality in patients with cirrhosis using cox regression model

\begin{tabular}{|c|c|c|}
\hline & OR $(95 \% \mathrm{CI})$ & $p$ \\
\hline \multicolumn{3}{|l|}{ MODEL1:AUC( 95\%CI):0.827(0.748-0.905) } \\
\hline Age & $1.096(1.058-1.135)$ & $<0.001$ \\
\hline WBC count & $1.126(1.054-1.202)$ & $<0.001$ \\
\hline INR & $5.890(2.852-12.164)$ & $<0.001$ \\
\hline \multicolumn{3}{|l|}{ MODEL2 :AUC( 95\%CI):0.873(0.817-0.930) } \\
\hline Age & $1.069(1.038-1.103)$ & $<0.001$ \\
\hline MELD Score & $1.190(1.118-1.267)$ & $<0.001$ \\
\hline \multicolumn{3}{|l|}{ MODEL3: AUC( 95\%CI):0.896(0.843-0.948) } \\
\hline Age & $1.064(1.030-1.098)$ & $<0.001$ \\
\hline MELD Score & $1.157(1.086-1.233)$ & $<0.001$ \\
\hline KDIGO criteria & $2.065(1.410-3.023)$ & $<0.001$ \\
\hline \multicolumn{3}{|l|}{ MODEL4: AUC( 95\%CI):0.920(0.881-0.958) } \\
\hline Age & $1.045(1.011-1.079)$ & 0.009 \\
\hline MELD Score & $1.156(1.087-1.230)$ & $<0.001$ \\
\hline Delta-sCr system & $2.911(2.007-4.223)$ & $<0.001$ \\
\hline \multicolumn{3}{|c|}{$\begin{array}{l}\text { OR, Odds Ratio; 95\%CI, 95\% confidence intervals; WBC, White blood cell; DM, } \\
\text { Diabetes mellitus; MELD, Model for End Stage Liver Disease; sCr , serum } \\
\text { creatinine; INR, International normalized ratio; KDIGO, Kidney Disease } \\
\text { Improving Global Outcomes; AUC, area under ROC curve }\end{array}$} \\
\hline
\end{tabular}

$\mathrm{OR}=2.065, p<0.001$ ) and 'Delta-sCr': OR=2.911, $p<0.001$ ), when included respectively in Model 2, Model 3 and 4).

ROC curve analysis was performed to evaluate the four models in predicting 1-year mortality. As shown in Table 2, the areas under the curve (AUC) for Model 1-4 were respectively $0.827,0.873,0.896$ and 0.920 . The results indicated that Model 4 including Age, MELD score and 'Delta-sCr' system had a better predictive value for 1-year mortality in cirrhotic patients ( $p=0.01, p=0.04, p=0.16$ ) in comparison with Model 1, 2 or 3 respectively.

Comparison of the prognostic value of KDIGO criteria vs 'Delta-sCr' system

Kaplan-Meier analysis and log-rank test were constructed to determine the differences in survival situation of AKI stages according to the two methods. Significant differences were found among the survival curves according to the 'Delta-sCr' system ( $p=0.023, p<0.001$, $p=0.016$, resp.).However, with the KDIGO criteria, the difference was only significant between AKI stages 1 and $3(p=0.032)$, not between AKI stage 1 and 3 and between stage 2 and 3 ( $p=0.639$ and $p=0.113$, resp.) (Fig. 1 ).

Compared with the original classification by the KDIGO criteria, the 'Delta-sCr' system reclassified 34 of 333 patients $(10.21 \%)$ to higher AKI stages, and 16 patients $(4.80 \%)$ to lower AKI stages. The total reclassification was hence 15.02\% (50/333).The 'Delta-sCr' system incorrectly downreclassified 3 of 31 deaths (9.68\%), and incorrectly up-reclassified 25 of 302 survivors (8.28\%). Though not very statistically significant in AUC comparison $(p=0.106)$, the 'Delta-sCr' system provided a modest improvement in classification over the KDIGO criteria with an NRI of $28.7 \%$ (95\% CI 12.1-42.5\%, $p<0.001$ ) and IDI of $7.5 \%(95 \%$ CI $0.7-16 \%, p=0.03$ ), suggesting that the risk levels for individuals reclassified by 'Delta-sCr' system might be more accurate to predict long-term risk mortality (Table 3).

The two methods classifying AKI according to KDIGO criteria and 'Delta-sCr' system were also assessed by calculating sensitivity, specificity, positive likelihood ratio(LR+)and negative likelihood ratio(LR-) for 1-year mortality as an outcome. The 'Delta-sCr' system delivered both increased specificity and LR+ at a cost of sensitivity or LR-, when classifying AKI stage 2 and 3. Whereas classifying AKI stage 1, the 'Delta-sCr' system exhibited improved sensitivity, with some loss of specificity (Table4). 


\section{Kidney Blood Pressure Research}

\section{Discussion}

The present study was conducted to investigate the performance of the 'DeltasCr' approach to stage AKI for predicting long-term outcomes, compared with the KDIGO criteria. To the best of our knowledge, this is the first study using the new approach to classification of AKI in cirrhotic patients. The diagnosis of ARF in cirrhosis is traditionally made by a $50 \%$ increase in $\mathrm{sCr}$ with setting a fixed sCr threshold of $\geq 1.5 \mathrm{mg} / \mathrm{dl}$ [14]. However, final consensus has not been reached yet on classification of cirrhosis-AKI. In the last decade, several attempts have been made. In 2002, the ADQI Working Group developed RIFLE criteria for AKI. Subsequent evidence that even small increase in $\mathrm{sCr}$ (as small as $0.3 \mathrm{mg} / \mathrm{dl}$ ) also has a negative impact on survival led to a modification of the RIFLE criteria called AKIN [3]. More recently, the KDIGO criteria have been developed to reach a consensus drawing consolidating elements of both RIFLE and AKIN on staging AKI [15]. Whether these criteria improve the traditional criterion (a final threshold value for $\mathrm{sCr}$ of $1.5 \mathrm{mg} / \mathrm{dl}$ ) in terms of a better prediction of mortality is still a matter of debate [16]. Piano et al. reported that the traditional criterion was more accurate than the AKIN criteria in the prediction of in-hospital mortality in patients with cirrhosis and ascites [17]. Furthermore, Fagundes et al. developed a 'new classification' that combined the AKIN criteria and traditional criterion in cirrhosis [9] (i) stage 1 with a final $\mathrm{sCr}$ of $\leq 1.5$ $\mathrm{mg} / \mathrm{dl}$; (ii) stage 1 with a final $\mathrm{sCr}$ of $>1.5 \mathrm{mg} / \mathrm{dl}$, and (iii) combined stages 2 and 3, providing a better prognostic stratification than AKIN criteria alone. Whereas, it has recently been challenged that even
Table 3. Log-rank tests refer to statistically significant differences between stages stratified by KDIGO and Delta-sCr system

\begin{tabular}{lcccc}
\hline & \multicolumn{5}{c}{ Log Rank- $p$} \\
\hline KDIGO critiria & AKI staging & 0 & $1 / \mathrm{I}$ & $2 / \mathrm{II}$ \\
& 0 & - & - & - \\
& 1 & $<0.001$ & - & - \\
& 2 & $<0.001$ & 0.639 & - \\
& 3 & $<0.001$ & 0.032 & 0.113 \\
\hline Delta-sCr system & 0 & - & - & - \\
& I & $<0.001$ & - & - \\
& II & $<0.001$ & 0.023 & - \\
& III & $<0.001$ & $<0.001$ & 0.016 \\
\hline
\end{tabular}

AKI, Acute Kidney Injury; KDIGO, Kidney Disease Improving Global Outcomes; sCr , serum creatinine

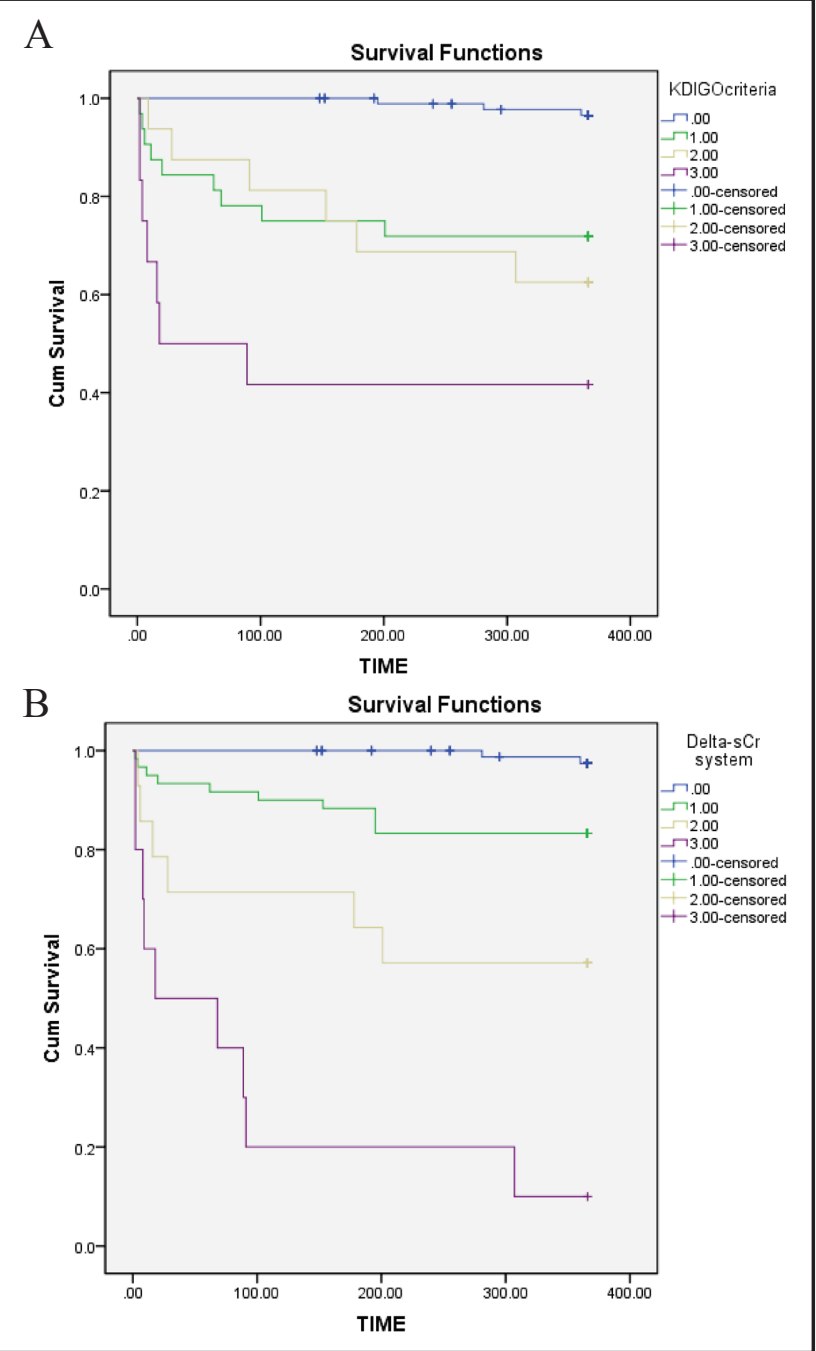

Fig. 1. Kaplan-Meier graphs for1-year survival stratified by KDIGO criteria (Figure 1A) and Delta-sCr system (Figure 1B) of AKI. 


\section{Kidney Blood Pressure Research}

Table 4. Accuracy of KDIGO criteria and Delta-sCr system in prediction of 1-year mortality

\begin{tabular}{lcccccccccc}
\hline & $\begin{array}{c}\text { AKI } \\
\text { staging }\end{array}$ & $\begin{array}{c}\text { Sensitivity } \\
(\%)\end{array}$ & $\begin{array}{c}\text { Specificity } \\
(\%)\end{array}$ & LR+ & LR- & $\begin{array}{c}\text { AUC } \\
(95 \% \mathrm{CI})\end{array}$ & $p$ & $\begin{array}{c}\text { IDI(\%) } \\
(95 \% \mathrm{CI})\end{array}$ & $p$ & $\begin{array}{c}\text { NRI(\%) } \\
(95 \% \mathrm{CI})\end{array}$ \\
\hline KDIGO & 1 & 29.03 & 92.38 & 3.81 & 0.77 & 0.803 & & & & \\
critiria & 2 & 19.35 & 96.67 & 5.85 & 0.83 & $(0.756-0.844)$ & & & \\
& 3 & 54.84 & 95.70 & 12.74 & 0.47 & & 0.108 & $(0.7-16.0)$ & 0.03 & $(12.1-42.5)$ \\
Delta-sCr & I & 32.26 & 83.44 & 1.95 & 0.81 & 0.844 & & & \\
system & II & 19.35 & 97.35 & 7.30 & 0.83 & $(0.801-0.882)$ & & & \\
\hline
\end{tabular}

AKI, Acute Kidney Injury; KDIGO, Kidney Disease Improving Global Outcomes; $\mathrm{SCr}$, serum creatinine; LR+, positive likelihood ratio; LR-, negative likelihood ratio; AUC, area under ROC curve; NRI, net reclassification improvement; IDI, integrated discrimination improvement; 95\% CI, 95\% confidence intervals

"mild" AKI in cirrhotic patients whose peak sCr remained below $1.5 \mathrm{mg} / \mathrm{dl}$ had a surprisingly higher mortality rate compared to those without AKI, indicating that the 'new classification' proposed by Fagundes et al. did not improve the prognostic assessment of patients with cirrhosis $[18,19]$. Therefore, it seems all of these various criteria are not that perfect, still requiring further refinement. At present, a number of studies have reported the absolute changes in $\mathrm{sCr}$ appear to be more accurate for estimating prognosis of AKI than the relative changes in $\mathrm{sCr}$, on which the cut-offs of AKIN or KDIGO criteria are mainly based. Those studies performed in general hospitalized patients and patients with acute myocardial infarction, however, the information on cirrhosis is lacking.

In our study, both staging methods (KDIGO criteria and 'Delta-sCr' system) were independent risk factors for mortality, however, compared to the 'Delta-sCr' system, the KDIGO criteria revealed lower OR or AUC value. And the KDIGO criteria showed no significant difference between AKI stage 1 and 3 and between stage 2 and 3. The new approach of 'Delta-sCr' system modestly improved the classification accuracy by IDI and NRI analysis, superior to the KDIGO criteria in cirrhosis. Further analysis showed that when classifying stage I, the 'Delta-sCr' system showed higher sensitivity to small sCr increases, which did not yet achieved the KDIGO criteria but might still yield a poor prognosis, providing a wider therapeutic window. This approach could be important in the early detection of mild renal injury for the potential to improve management, more closely monitoring, halting the process of renal injury. Also, the new expanded diagnostic criteria could help to identify AKI, which would be missed by KDIGO criteria for delayed sCr repeated assay, and sustained progressive kidney injury, which had an increase in $\mathrm{sCr}$ that was less rapid than what the KDIGO criteria required [20]. While classifying AKI stage II and III, the 'Delta-sCr' system showed higher specificity and LR+, avoiding undue changes in management. In our study, AKI were classified when they first fulfilled KDIGO criteria, but reclassified by 'Delta-sCr' in a dynamic context, especially when progressing to more severe one. It is reported that patient survival is not only impacted by the stage of renal dysfunction but also by the progression of renal dysfunction on follow-up [21]. Taking into account that, it may be logical this new approach could be a more sensitive, accurate and simple way for prognostic assessment and targeted interventions.

Besides the new staging approach we proposed, there are several strengths to this study. First, we use of a relatively non-selected cirrhotic patient population with ascites or not, and a wide range of comorbidities, except cancer and ESRD, providing the potential generalization of the new staging approach. Second, in previous studies, the admission sCr were commonly used as the baseline value, which would have obscured the presence or severity of AKI, and the mortality risk associated with various stages of AKI [15]. IAC and ADQI defined baseline $\mathrm{sCr}$ as the stable $\mathrm{sCr}$ value within the last 3 months before admission [5]. However, in clinical practice, this timeframe seems unfeasible in most cases. So we had prolonged the timeframe to 1 year, which considered to be most closely approximating nephrologist-adjudicated basal SCr values [22, 23]. In our study, most of the patients (69.67\%) used the pre-hospitalized sCr. And the admission sCr were only used in those (30.33\%) without the pre-hospitalized sCr. Third, the studied population was from China. There are obvious differences in the etiology 


\section{Kidney \\ Blood Pressure Research}

of cirrhosis between the East and West. In Western societies, alcohol is the main etiology of cirrhotic patients. In contrast, in the Asia-Pacific region, the majority of cirrhosis is caused by HBV [24]. Therefore, it was interesting to investigate whether the current KDIGO AKI staging also applies in the Asia-Pacific region.

\section{Limitations}

Our study also has some limitations that should be acknowledged. First, it was a singlecentre retrospective study. Being observational in nature, we did not have daily sCr values for all patients, which might predispose to bias and residual confounding. In fact, in daily routine, there is less attention to the renal function paid by the hepatologists. Repeated measures of $\mathrm{sCr}$ were not always available, especially within 48 hours for defining AKI by KDIGO criteria. A multicenter prospective study capturing real-time laboratory and clinical characteristics are needed for further verification. Second, although the new AKI staging approach of 'Delta-sCr' system herein did well in long-term risk prediction in cirrhosis, the cut-offs we selected for each 'Delta-sCr' stage were retrospective data derived. So, it is very important to evaluate the 'Delta-sCr' system and its cut-offs with larger, external validation data set. Third, $\mathrm{sCr}$ is no doubt an inexpensive and the most practical biomarker of renal function. However, it is well recognized that $\mathrm{sCr}$ is a neither sensitive nor specific marker of kidney injury for cirrhotic patients [25]. As a consequence, GFR is also inaccurate in cirrhosis, since it is calculated based on sCr measurement [26]. Recently, many novel biomarkers have been explored for early diagnosis of AKI, including neutrophil gelatinase-associated lipocalin (NGAL), interleukin-18 (IL-18) and kidney injury molecule-1 (KIM-1), and Toll-like receptor 4 (TLR4) [27-31]. Moreover, it is not likely that AKI alone is adequate for risk stratification in patients with cirrhosis [32-34]. Our study also revealed that age and MELD score predicted survival in cirrhosis as well. Thus, the two prognostic factors were combined in the final model (Model 4), leading to the incremental value of the 'Delta-sCr' system in prediction of mortality (Model 4: AUC=0.920, 'Delta-sCr': AUC=0.844, $p=0.006$ ).

\section{Conclusion}

In summary, the 'Delta-sCr' system, based on absolute increases in $\mathrm{sCr}$, appeared to better stratify cirrhosis-AKI and predict long-term mortality risk than the current KDIGO criteria. However, optimization of the cut points for the new 'Delta-sCr' system still needs to be prospectively validated. Moreover, novel biomarkers and other prognostic factors related to cirrhosis should be considered to combine with AKI classification for improved predictive ability.

\section{Disclosure Statement}

There are no conflicts of interest associated with this work.

\section{Acknowledgments}

This study was supported in part by a project from Medical Science Research Foundation of Zhejiang Province (Grant No. 201336474, to QL) and Ningbo city Natural Science Foundation (Grant No. 2013A610266, to FFZ). The funders had no role in study design, data collection and analysis, decision to publish, or preparation of the manuscript. 


\section{Kidney \\ Blood Pressure Research}

\section{References}

1 Karvellas CJ, Durand F, Nadim MK: Acute Kidney Injury in Cirrhosis. Crit Care Clin 2015;31:737-750.

2 Belcher JM, Parikh CR, Garcia-Tsao G: Acute kidney injury in patients with cirrhosis: perils and promise. Clin Gastroenterol Hepatol 2013;11:1550-1558.

3 Wong F: Definition and Diagnosis of Acute Kidney Injury in Cirrhosis. Dig Dis 2015;33:539-547.

-4 Fede G, D'Amico G, Arvaniti V, Tsochatzis E, Germani G, Georgiadis D, Morabito A, Burroughs AK: Renal failure and cirrhosis: a systematic review of mortality and prognosis. J Hepatol 2012;56:810-818.

5 Angeli P, Gines P, Wong F, Bernardi M, Boyer TD, Gerbes A, Moreau R, Jalan R, Sarin SK, Piano S, Moore K, Lee SS, Durand F, Salerno F, Caraceni P, Kim WR, Arroyo V, Garcia-Tsao G: Diagnosis and management of acute kidney injury in patients with cirrhosis: revised consensus recommendations of the International Club of Ascites. J Hepatol 2015;62:968-974.

-6 Egerod LM, Gluud LL, Krag A: Acute kidney injury and hepatorenal syndrome in cirrhosis. J Gastroenterol Hepatol 2015;30:236-243.

7 Allegretti AS, Ortiz G, Wenger J, Deferio JJ, Wibecan J, Kalim S, Tamez H, Chung RT, Karumanchi SA, Thadhani RI: Prognosis of Acute Kidney Injury and Hepatorenal Syndrome in Patients with Cirrhosis: A Prospective Cohort Study. Int J Nephrol 2015;2015:108139.

-8 Belcher JM, Garcia-Tsao G, Sanyal AJ, Bhogal H, Lim JK, Ansari N, Coca SG, Parikh CR: Association of AKI with mortality and complications in hospitalized patients with cirrhosis. Hepatology 2013;57:753-762.

-9 Fagundes C, Barreto R, Guevara M, Garcia E, Sola E, Rodriguez E, Graupera I, Ariza X, Pereira G, Alfaro I, Cardenas A, Fernandez J, Poch E, Gines P: A modified acute kidney injury classification for diagnosis and risk stratification of impairment of kidney function in cirrhosis. J Hepatol 2013;59:474-481.

10 Berra G, Garin N, Stirnemann J, Jannot AS, Martin PY, Perrier A, Carballo S: Outcome in acute heart failure: prognostic value of acute kidney injury and worsening renal function. J Card Fail 2015;21:382-390.

11 Fox CS, Muntner P, Chen AY, Alexander KP, Roe MT, Wiviott SD: Short-term outcomes of acute myocardial infarction in patients with acute kidney injury: a report from the national cardiovascular data registry. Circulation 2012;125:497-504.

12 Wang HE, Jain G, Glassock RJ, Warnock DG: Comparison of absolute serum creatinine changes versus Kidney Disease: Improving Global Outcomes consensus definitions for characterizing stages of acute kidney injury. Nephrol Dial Transplant 2013;28:1447-1454.

13 KDIGO Clinical Practice Guideline for Acute Kidney Injury. Kidney Int Suppl 2012;2:2.

14 Wong F: The evolving concept of acute kidney injury in patients with cirrhosis. Nat Rev Gastroenterol Hepatol 2015;12:711-719.

15 Lameire N: The definitions and staging systems of acute kidney injury and their limitations in practice. Arab J Nephrol Transplant 2013;6:145-152.

16 Wong F: Treatment to improve acute kidney injury in cirrhosis. Curr Treat Options Gastroenterol 2015;13:235-248.

17 Piano S, Rosi S, Maresio G, Fasolato S, Cavallin M, Romano A, Morando F, Gola E, Frigo AC, Gatta A, Angeli P: Evaluation of the Acute Kidney Injury Network criteria in hospitalized patients with cirrhosis and ascites. J Hepatol 2013;59:482-489.

18 Bucsics T, Mandorfer M, Schwabl P, Bota S, Sieghart W, Ferlitsch A, Trauner M, Peck-RM, Reiberger T: Impact of acute kidney injury on prognosis of patients with liver cirrhosis and ascites: A retrospective cohort study. J Gastroenterol Hepatol 2015;30:1657-1665.

19 Tsien CD, Rabie R, Wong F: Acute kidney injury in decompensated cirrhosis. Gut 2013;62:131-137.

-20 Yang L, Xing G, Wang L, Wu Y, Li S, Xu G, He Q, Chen J, Chen M, Liu X, Zhu Z, Yang L, Lian X, Ding F, Li Y, Wang H, Wang J, Wang R, Mei C, Xu J, Li R, Cao J, Zhang L, Wang Y, Xu J, Bao B, Liu B, Chen H, Li S, Zha Y, Luo Q Chen D, Shen Y, Liao Y, Zhang Z, Wang X, Zhang K, Liu L, Mao P, Guo C, Li J, Wang Z, Bai S, Shi S, Wang Y, Wang J, Liu Z, Wang F, Huang D, Wang S, Ge S, Shen Q, Zhang P, Wu L, Pan M, Zou X, Zhu P, Zhao J, Zhou M, Yang L, Hu W, Wang J, Liu B, Zhang T, Han J, Wen T, Zhao M, Wang H, ISN AKF 0by25 China Consortiums: Acute kidney injury in China: a cross-sectional survey. Lancet 2015;386:1465-1471.

-21 Biyik M, Ataseven H, Biyik Z, Asil M, Çifçi S, Sayın S, Tonbul HZ, Demir A: KDIGO (Kidney Disease: Improving Global Outcomes) criteria as a predictor of hospital mortality in cirrhotic patients. Turk J Gastroenterol 2016;27:173-179. 


\section{Kidney \\ Blood Pressure Research}

-22 Siew ED, Ikizler TA, Matheny ME, Shi Y, Schildcrout JS, Danciu I, Dwyer JP, Srichai M, Hung AM, Smith JP, Peterson JF: Estimating baseline kidney function in hospitalized patients with impaired kidney function. Clin J Am Soc Nephrol 2012,7:712-719.

-23 Siew ED, Matheny ME, Ikizler TA, Lewis JB, Miller RA, Waitman LR, Go AS, Parikh CR, Peterson JF: Commonly used surrogates for baseline renal function affect the classification and prognosis of acute kidney injury. Kidney Int 2010,77:536-542.

-24 Kim TY, Kim DJ: Acute-on-chronic liver failure. Clin Mol Hepatol 2013;19:349-359.

25 Regner KR, Singbartl K: Kidney Injury in Liver Disease. Crit Care Clin 2016;32:343-355.

-26 Rosi S, Piano S, Frigo AC, Morando F, Fasolato S, Cavallin M, Gola E, Romano A, Montagnese S, Sticca A, Gatta A, Angeli P: New ICA criteria for the diagnosis of acute kidney injury in cirrhotic patients: can we use an imputed value of serum creatinine? Liver Int 2015;35:2108-2114.

27 Wong F, Murray P: Kidney damage biomarkers: Novel tools for the diagnostic assessment of acute kidney injury in cirrhosis. Hepatology 2014;60:455-457.

28 Belcher JM: Acute Kidney Injury in Liver Disease: Role of Biomarkers. Adv Chronic Kidney Dis 2015;22:368-375.

-29 Shah N, Mohamed FE, Jover-Cobos M, Macnaughtan J, Davies N, Moreau R, Paradis V, Moore K, Mookerjee R, Jalan R: Increased renal expression and urinary excretion of TLR4 in acute kidney injury associated with cirrhosis. Liver Int 2013;33:398-409.

-30 Zhou F, Luo Q, Wang L, Han L: Diagnostic value of neutrophil gelatinase-associated lipocalin for early diagnosis of cardiac surgery-associated acute kidney injury: a meta-analysis. Eur J Cardiothorac Surg 2016;49:746-755.

31 Luo Q, Zhou F, Dong H, Wu L, Chai L, Lan K, Wu M: Implication of combined urinary biomarkers in early diagnosis of acute kidney injury following percutaneous coronary intervention. Clin Nephrol 2013;79:8592.

-32 Hoste EA, Kashani K, Gibney N, Wilson FP, Ronco C, Goldstein SL, Kellum JA, Bagshaw SM,15 ADQI Consensus Group: Impact of electronic-alerting of acute kidney injury: workgroup statements from the 15(th) ADQI Consensus Conference. Can J Kidney Health Dis 2016;3:10.

-33 Angeli P, Rodriguez E, Piano S, Ariza X, Morando F, Solà E, Romano A, García E, Pavesi M, Risso A, Gerbes A, Willars C, Bernardi M, Arroyo V, Ginès P, CANONIC Study Investigators of EASL-CLIF Consortium: Acute kidney injury and acute-on-chronic liver failure classifications in prognosis assessment of patients with acute decompensation of cirrhosis. Gut 2015;64:1616-1622.

34 Arroyo V: Acute kidney injury (AKI) in cirrhosis: Should we change current definition and diagnostic criteria of renal failure in cirrhosis? J Hepatol 2013;59:415-417. 